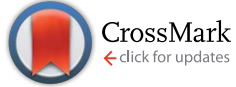

Cite this: RSC Adv., 2015, 5, 4502

Received 26th September 2014 Accepted 4th December 2014

DOI: 10.1039/c4ra11289j

www.rsc.org/advances

\section{Self-assembly of a cholesteryl-modified nucleoside into tubular structures from giant unilamellar vesicles $\uparrow$}

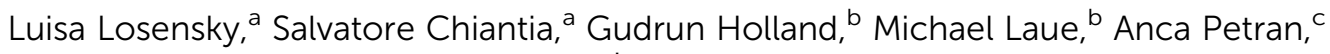 \\ Jürgen Liebscher ${ }^{c}$ and Anna Arbuzova*a
}

\begin{abstract}
We recently reported the formation of micro- and nanometer thick tubules in a binary system of cholesteryl-modified aminouridine and a phosphatidylcholine in aqueous solution upon cooling from $70{ }^{\circ} \mathrm{C}$ to room temperature (Pescador et al., Chem. Commun, 2010, 46, 5358). To better understand the first steps of the tubular self-assembly and the role of the phospholipid, we investigated now morphological changes of phosphatidylcholine giant unilamellar vesicles upon incorporation of the conjugate at room temperature. We observed formation of needle-like structures organized in rosettes or bundles within hours to days depending on the mole fraction of the conjugate and preparation technique. Transmission electron microscopy revealed that the needle-like structures were 100-200 nm thick tubules with the inner lumen diameters of $15-40 \mathrm{~nm}$. The tube self-assembly occurred at room temperature, which is important for further development of biomedical applications.
\end{abstract}

\section{Introduction}

Self-assembly of amphiphilic molecules into micelles, vesicles, sheets, or tubular structures are governed by an interplay of hydrophobic interactions, hydrogen bonding, stacking, structure (form, chirality) of the molecules, and interaction with the solvent. ${ }^{1-5}$ The pseudo one-dimensional needle-like and tubular structures are of particular interest, as they are not only found in nature, e.g. cholesterol (Chol) needle-like crystals in bile and atherosclerotic plaques, ${ }^{6,7}$ but can be also used for different applications, e.g. as templates for inorganic nanotubules and wires, drug-delivery systems, single-molecule sensing, cleaning of water, ${ }^{8-10}$ and for structuring of edible oils to margarine. ${ }^{11}$

Different mechanisms were suggested for the formation of the tubular structures: rolling of membrane sheets, fusion of vesicles, growth of helical structures in width or decreasing pitch. ${ }^{12,13}$ However, the mechanism of the tubular structure assembly is still not well understood; especially as different assembly mechanisms were found in binary systems depending on the molar ratio of the components. ${ }^{14}$

Both cholesterol and nucleoside derivatives were reported to self-assemble into needles, helices, tubular structures, or

\footnotetext{
${ }^{a}$ Humboldt-Universität zu Berlin, Institut für Biologie, Invalidenstr. 42, 10115 Berlin, Germany. E-mail: arbuzova@cms.hu-berlin.de

${ }^{b}$ Robert Koch Institute, ZBS 4, Nordufer 20, 13353 Berlin, Germany

${ }^{c}$ National Institute of Research and Development for Isotopic and Molecular Technologies, Donath 65-103, RO-400293 Cluj-Napoca, Romania

$\dagger$ Electronic supplementary information (ESI) available. See DOI: $10.1039 / \mathrm{c} 4 \mathrm{ra} 11289 \mathrm{j}$
}

vesicles. $^{\mathbf{1 0 , 1 5 , 1 6}}$ Sharp needle-like crystals, micrometer-sized helices, and tubules mostly made of cholesterol were found in both patient samples and model bile mixtures. ${ }^{6,17,18} \mathrm{~A}$ native bile salt component, sodium lithocholate, was found to form single molecular layer tubes with diameters about $50 \mathrm{~nm} .{ }^{19}$ Lithocholic acid formed tubules in aqueous solution of $\mathrm{pH} 12$ with a continuous growth of the tubules as long as vesicles were present. ${ }^{20}$ Chemically modified bile salts, e.g. a sodium cholate derivative, self-assembled into tubules of about $450 \mathrm{~nm}$ diameter and length up to $7 \mu \mathrm{m}$ when heated above $40{ }^{\circ} \mathrm{C}^{21,22}$ Uracilmodified cholesterol was reported to form fibers/columnar structures in organic solvents. However, only precipitation was observed in aqueous solutions. ${ }^{23}$ Other cholesteryl conjugates of nucleosides, e.g. acyclovir and zidovudine commonly used as antiviral drugs, formed $100-200 \mathrm{~nm}$ size bilayer vesicles in aqueous solutions when injected from THF. ${ }^{24}$

We have reported on the micro- and nano-tubule formation from $2^{\prime}-N$-(2-(cholesteryl)-succinyl)-2'-deoxy-2'-aminouridine (CholAU, Fig. 1A) with 1,2-dioleoyl-sn-glycero-3-phosphocholine (DOPC) upon cooling of a $30: 70$ CholAU : DOPC (mol\%) aqueous suspension from $70{ }^{\circ} \mathrm{C}$ to room temperature. ${ }^{25}$ CholAU combines selfassembly properties of cholesterol (hydrophobicity, chirality) and uridine (stacking, hydrogen bonding). To understand the first steps of the tubular self-assembly and the role of the phospholipid, we now investigated morphological changes of phosphatidylcholine (PC) and CholAU binary system upon the incorporation of CholAU into PC giant unilamellar vesicles (GUVs) using several approaches: (i) from a complex with methyl- $\beta$-cyclodextrin (m $\beta \mathrm{CD}$-CholAU) to assure incorporation of monomeric CholAU into the lipid membrane; (ii) from DMSO 

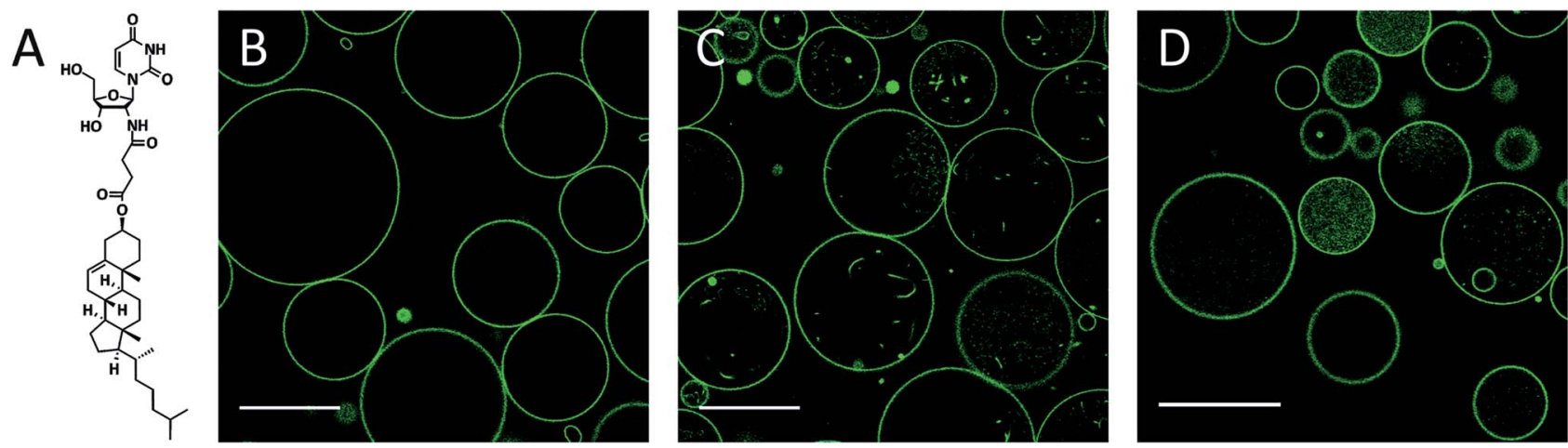

Fig. 1 (a) Chemical structure of the conjugate $2^{\prime}-N$-(2-(cholesteryl)-succinyl)-2'-deoxy-2'-aminouridine (CholAU). (b-d) NBD fluorescence images: DMOPC: NBD-DMPE 99.2 : 0.8 (molar ratio) GUVs at times 0 s and 30 min after exchange of $5 \mathrm{mM}$ glucose with $2.5 \mathrm{mM} \mathrm{m} \beta C D-C h o l A U$ solution (b) and (c), respectively. (d) GUVs $30 \mathrm{~min}$ after exchange of the solution to $5 \mathrm{mM} \mathrm{m} \beta C D$. Scale bars correspond to $50 \mu \mathrm{m}$.

solution of CholAU; and (iii) by preparation of GUVs starting with a CholAU-PC mixture in organic solvent for a better control of the incorporated amount.

GUVs are widely used models of cell membranes and consist of unilamellar vesicles with typical dimensions in the $\mu \mathrm{m}$-range. Their size and curvature resemble those of actual cellular plasma membranes and, moreover, are compatible with the application of optical microscopy. ${ }^{26,27}$ GUVs have been successfully used in the last years to investigate lipid-protein interactions, membrane protein lateral organization, ${ }^{28}$ membrane curvature and tubule formation, ${ }^{29-31}$ lipid-lipid interaction, e.g. PC-Chol interaction, ${ }^{32-34}$ and specific domain partitioning of lipid-modified conjugates. ${ }^{35-37}$

The self-assembly of the tubular structures from GUVs containing CholAU was investigated by fluorescence and electron microscopy. The results provide information on the role of phospholipid vesicles in the tubular self-assembly and in how far the uridine moiety of CholAU changes the interaction with lipid membranes in comparison with published results obtained with cholesterol.

\section{Experimental}

\section{Chemicals}

1,2-Dimyristoleoyl-sn-glycero-3-phosphocholine (DMoPC) and 1,2-dimyristoyl-sn-glycero-3-phosphoethanolamine- $N$-(7-nitro-21,3-benzoxadiazol-4-yl) (ammonium salt), (NBD-DMPE) were purchased from Avanti Polar Lipids (Alabaster, AL). Methyl- $\beta$ cyclodextrin ( $\mathrm{m} \beta \mathrm{CD}, 1.6-2.0 \mathrm{~mol} \mathrm{CH}_{3}$ per unit anhydroglucose) and sucrose were purchased from Sigma-Aldrich (Taufkirchen, Germany). Chloroform and methanol were supplied from Merck (Darmstadt, Germany). Glucose was obtained from Roth (Karlsruhe, Deutschland) and $2^{\prime}-N$-(2-(cholesteryl)-succinyl)-2'desoxy-2'-aminouridine (CholAU) was synthesized as described previously. ${ }^{25}$

\section{Giant unilamellar vesicles}

Giant unilamellar vesicles (GUVs), $30 \mathrm{nmol}$ total lipid, were formed from 99.2: 0.8 DMoPC : NBD-DMPE (molar ratio) by electroformation $^{38}$ in $5 \mathrm{mM}$ sucrose solution using homemade flow chambers with an observation volume of about $300 \mu \mathrm{L}$ as described previously. $^{39}$ For fluorescence microscopy, GUVs remained attached to the indium tin oxide (ITO) coated slides allowing efficient exchange of the solvent. For electron microscopy, GUVs were grown on platinum wires in $300 \mathrm{mOsm}$ sucrose solution in homemade chambers. Upon formation, they were collected and carefully pipetted into a 300 mOsm glucose solution. For the preparation of the DMoPC : CholAU : NBD-DMPE mixtures in chloroform-methanol as solvent, the total lipid amount was kept at $30 \mathrm{nmol}$, using 40, 20 and $10 \mathrm{~mol} \%$ CholAU (molar ratios: $59.2: 40: 0.8, \quad 79.2: 20: 0.8$ and $89.2: 10: 0.8$ of DMoPC : CholAU : NBD-DMPE).

\section{Preparation of $\mathbf{m} \beta C D-C h o l A U$ complex}

Methyl- $\beta$-cyclodextrin (m $\beta \mathrm{CD}$ ) and CholAU were mixed shortly before addition to DMoPC GUVs or overnight. For the shortly mixed complex, $0.8 \mu \mathrm{mol}$ of CholAU were dissolved in $\mathrm{CHCl}_{3}-\mathrm{MeOH}(5: 1)$ in glass vials and dried using nitrogen gas. The CholAU-film was dispersed in $100 \mu \mathrm{L}$ of $25 \mathrm{mM}$ aqueous solution of $\mathrm{m} \beta \mathrm{CD}$, shortly sonicated and incubated in a shaker for $30 \mathrm{~min}$ at room temperature. For the overnight mixed complex, $0.8 \mu \mathrm{mol}$ of CholAU and $2.5 \mu \mathrm{mol}$ of $\mathrm{m} \beta \mathrm{CD}$ were dissolved in $150 \mu \mathrm{L} \mathrm{MeOH}$, vortexed at room temperature, dried using nitrogen stream and kept at high vacuum for

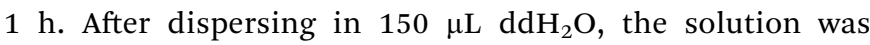
sonicated for $3 \mathrm{~min}$ and incubated in a shaker at $37^{\circ} \mathrm{C}$ overnight. ${ }^{40}$ The solution in the GUV chambers was exchanged to a $2.5 \mathrm{mM}$ solution of $\mathrm{m} \beta \mathrm{CD}-\mathrm{CholAU}$ (calculated molar ratio $3: 1$ ) by injecting $600 \mu \mathrm{L}$ through the chamber. As a control experiment the influence of $\mathrm{m} \beta \mathrm{CD}$ on GUVs was tested. For this, $600 \mu \mathrm{L}$ of $5 \mathrm{mM} \mathrm{m} \beta \mathrm{CD}$ were injected into the chamber. The assembly process of needle-like structures was qualitatively similar independently of the duration of the $\mathrm{m} \beta \mathrm{CD}-\mathrm{CholAU}$ mixing step.

\section{Addition of CholAU in DMSO}

$20 \mu \mathrm{L}$ of $1 \mathrm{mg} \mathrm{mL}{ }^{-1}$ CholAU solution in DMSO were mixed with $580 \mu \mathrm{L}$ water, yielding a final CholAU concentration of $47 \mu \mathrm{M}$. This solution was immediately injected into the flow chamber 
containing $100 \mu \mathrm{M}$ DMoPC : NBD-DMPE GUVs (i.e. $30 \mathrm{nmol}$ in the $300 \mu \mathrm{L}$ chambers, see above). Incorporation of all CholAU into GUVs would correspond to $\sim 30 \mathrm{~mol} \%$ of CholAU of total lipid.

\section{Confocal microscopy}

Olympus Fluoview FV-1000MPE, Inverted Laser Scanning microscope, Olympus (Tokyo, Japan) was used with a $488 \mathrm{~nm}$ Argon laser and $60 \times / 1.2$ Water UPlanSApo objective. Laser light was directed to a sample and separated from emitted light with a dichroic mirror (DM 405/488/559/635), NBD fluorescence was recorded between 500 and $600 \mathrm{~nm}$ with $4 \mu \mathrm{s} /$ pixel exposure. Note, low level of irradiation is important, as strong irradiation induces GUVs shape changes, lipid oxidation, and domain formation. ${ }^{41}$ Contrast of the fluorescence microscopy images was linearly enhanced.

\section{Mass spectrometry}

$\mathrm{CD}$-CholAU complexes were prepared as previously described $^{42}$ using acetonitrile instead of $\mathrm{MeOH}$. The formation of complexes was shown by ESI-FTICRMS (electrospray ionization-Fourier transform ion cyclotron resonance mass spectrometry). Samples were measured on an Ionspec QFT-7, Varian Inc. (Lake Forest, CA), equipped with a $7 \mathrm{~T}$ superconducting magnet and a Micromass Z-Spray ESI-Source, Waters Co. (Saint-Quentin, France). Solvent flow rate was adjusted to $4 \mu \mathrm{L} \min ^{-1}$, spray voltage was set to $3.8 \mathrm{kV}$. All other parameters were adjusted for an optimized detection of $[\beta C D-C h o l A U-H]^{-}$and $\left[\beta \mathrm{CD}_{2}-\mathrm{CholAU}-2 \mathrm{H}\right]^{2-}$ complexes. $^{43}$

\section{Negative staining transmission electron microscopy (TEM)}

To allow the transfer of the samples onto electron microscopy grids, DMoPC : NBD-DMPE 99.2 : 0.8 (molar ratio) GUVs were grown on platinum wires in 300 mOsm sucrose and transferred into 300 mOsm glucose solution in plastic tubes. CholAU was incubated with $\mathrm{m} \beta \mathrm{CD}$ either $30 \mathrm{~min}$ or overnight as described above. $50 \mu \mathrm{L}$ of $100 \mu \mathrm{M}$ GUVs were added to $250 \mu \mathrm{L}$ of 300 mOsm glucose solution. Then $50 \mu \mathrm{L}$ of the $2.5 \mathrm{mM} \mathrm{m} \beta \mathrm{CD}-$ CholAU solution were added. For negative staining TEM glucose was removed by sedimentation and resuspension with distilled water. A small volume $(5 \mu \mathrm{L})$ of the suspension was adsorbed at Pioloform coated, carbon stabilized and alcian blue treated (Fluka, $1 \%$ in $1 \%$ acetic acid in water) copper grids. Excess suspension was removed by gently blotting with a wetted filter paper and the sample was stained with $0.25 \%$ aqueous uranyl acetate. The samples were analyzed using a TEM 902 (Zeiss, Oberkochen; Germany) operated at $80 \mathrm{kV}$. Micrographs were recorded with a slow scan CCD - camera (Proscan, Scheuring, Germany). Dimensions of 96 tubules from 4 samples were measured using ImageJ.

\section{Results and discussion}

\section{Formation of needle-like structures upon incubation of DMoPC GUVs with $\mathbf{m} \beta C D-C h o l A U$ complex}

DMoPC GUVs formed in $5 \mathrm{mM}$ glucose by electroformation on indium tin oxide (ITO) coated slides were $10-50 \mu \mathrm{m}(n=68)$ in diameter and had almost no membrane structures inside (Fig. 1B). The glucose solution was exchanged with a $2.5 \mathrm{mM}$ aqueous solution of CholAU pre-incubated with methyl- $\beta$ cyclodextrin $(\mathrm{m} \beta \mathrm{CD})$. Water-soluble cyclodextrins are capable of complex formation with lipids, e.g. $\mathrm{m} \beta \mathrm{CD}$ was used to produce asymmetric liposomes, ${ }^{\mathbf{3 9}, 40}$ to extract Chol from membranes ${ }^{44}$ and to change Chol concentration in cells. ${ }^{45} \mathrm{We}$ used $\mathrm{m} \beta \mathrm{CD}$ to solubilize CholAU and deliver it to lipid bilayers in an aqueous solution, which is important when working with pre-formed lipid membranes, e.g. GUVs. Mass spectrometry measurements revealed $1: 1$ and $2: 1$ ([ $[\beta \mathrm{CD}-\mathrm{CholAU}-\mathrm{H}]^{-}$and $\left.\left[\beta \mathrm{CD}_{2}-\mathrm{CholAU}-2 \mathrm{H}\right]^{2-}\right)$ complexes of the structurally and functionally related unmethylated $\beta \mathrm{CD}$ and CholAU.

Membrane tubules and smaller vesicles were detected in most GUVs after incubation with $\mathrm{m} \beta \mathrm{CD}-\mathrm{CholAU}$ for $30 \mathrm{~min}$ (Fig. 1C). These invaginations were probably formed due to the incorporation of CholAU first into the outer membrane of the vesicles. Vesicle shape deformations and budding/tubule formation were reported upon a local change of the membrane composition or environment. ${ }^{30,31,46-49}$ For example, tubular invaginations of $240 \mathrm{~nm}$ diameter were observed upon aqueous phase separation (osmotic stress) in DOPC/ganglioside GUVs. ${ }^{50}$ Although cholesterol was reported to flip quickly and spontaneously into the inner leaflet with a flip-flop halftime in the order of 1 min for liquid disordered membranes, ${ }^{51,52}$ it is not known yet how fast CholAU can cross the membrane. Note after incubation of GUVs with $\mathrm{m} \beta \mathrm{CD}$ alone, some GUVs revealed green fluorescence inside of the vesicles and occasionally budding/invaginations were also observed as shown in Fig. 1D. The $m \beta C D$ does not penetrate through the membrane of the GUVs, but, occasionally, some GUVs might be temporarily leaky, potentially resulting in $\mathrm{m} \beta \mathrm{CD}$ influx into the GUVs and extraction of the fluorescent dye. Then the GUV would reseal and the $m \beta C D$-dye complexes stay in the lumen of the vesicle, giving rise to the fluorescence inside some of the vesicles in control measurements as seen in Fig. 1D.

Although, at concentrations of $\mathrm{m} \beta \mathrm{CD}$ below $10 \mathrm{mM}$ no considerable extraction of phospholipids was reported, a variable extent of cyclodextrin-phospholipid interactions was observed in different studies. ${ }^{53}$ Hence, for a phospholipid with short acyl chains and low lipid/m $\beta C D$ ratio used, limited lipid extraction could occur also at a concentration of $\mathrm{m} \beta \mathrm{CD}$ below 5 $\mathrm{mM}$. Since DMoPC would be extracted only from the outer leaflet, the trans-bilayer mass misbalance might cause negative curvature, thus explaining the invagination of the bilayer that was occasionally observed in control samples.

Three hours after addition of the complex, compact optically dense fluorescent needle-like structures were observed as shown in Fig. 2A. The needle-like structures were organized in rosettes with a few vesicles in between on the glass surface, 

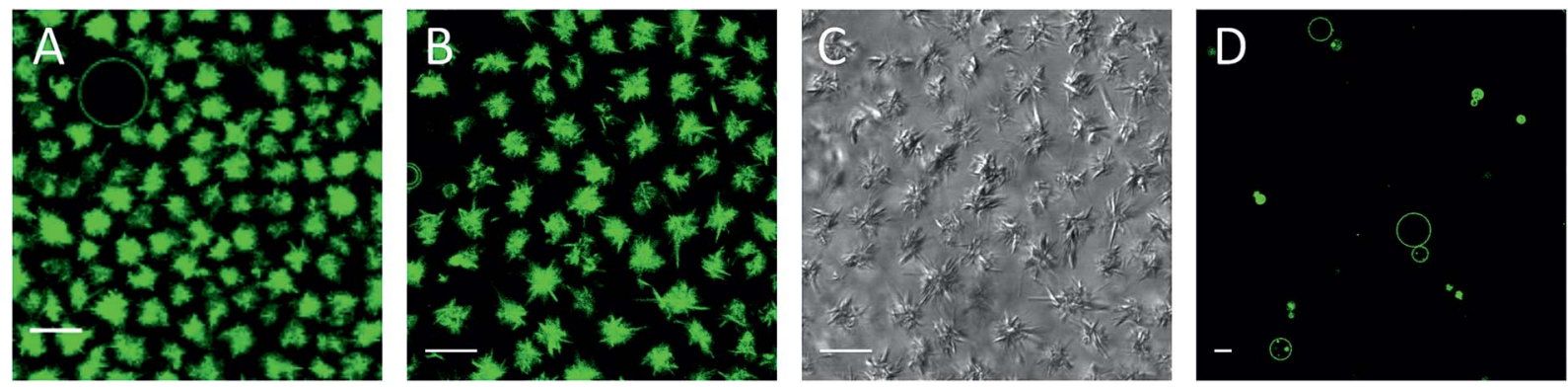

Fig. 2 Needle-like structures 3 hours (a) and one day (b and c) after addition of $2.5 \mathrm{mM} \mathrm{m \beta CD-CholAU} \mathrm{to} \mathrm{DMoPC} \mathrm{:} \mathrm{NBD-DMPE} 99.2$ : 0.8 (molar ratio) GUVs. ( $a, b$ and d) NBD fluorescence, (c) differential interference contrast (DIC) images, respectively. (d) Control: GUVs one day after exchange of the solution to $5 \mathrm{mM} \mathrm{m} \beta \mathrm{CD}$. Scale bars correspond to $10 \mu \mathrm{m}$.

whereas above the glass surface still more vesicles were found (not shown). We assume that upon incorporation of monomeric CholAU into the PC membrane, metastable structures were formed with a tendency for a phase separation leading further to the formation of stable needle-like structures. Sometimes, single needle-like structures covered with lipid bilayer structures were observed inside of the vesicles (see Fig. 5B). Needles grew larger, causing deformation of the vesicle shape and then the vesicle ruptured. Therefore, the number of GUVs decreased in time (hours). Similar rupture of vesicles caused by lysozyme crystals growth was reported previously. ${ }^{54}$ One day after $\mathrm{m} \beta \mathrm{CD}-$ CholAU addition, predominantly needle-like structures with some GUVs were observed (Fig. $2 \mathrm{~B}$ and C). 6 days after $\mathrm{m} \beta \mathrm{CD}-$ CholAU addition, almost no GUVs were left whereas the needlelike structures were mostly found on the glass surface organized into rosettes or bundles as well as in the solution above (not shown). In the control samples containing $5 \mathrm{mM} \mathrm{m} \beta \mathrm{CD}$ no needle-like structures were observed at any time (Fig. 2D, one day after solution exchange).

\section{Addition of CholAU to GUVs from DMSO solution also led to assembly of needle-like structures}

Alternatively, CholAU was solubilized in DMSO. This solvent was reported to have no or little effect on the bilayer structure and GUVs when added at low concentrations $(0.16-0.5 \mathrm{v} / \mathrm{v} \%) .^{55}$ We also observed no significant effect of DMSO addition on DMoPC GUVs (Fig. 3A); mostly unilamellar vesicles with average diameters of $10-40 \mu \mathrm{m}(n=78)$ were observed 1 hour after addition of $20 \mu \mathrm{L}$ DMSO in $580 \mu \mathrm{L}$ water to the flow chamber $(3.3 \mathrm{v} / \mathrm{v} \%)$. When a solution of $47 \mu \mathrm{M}$ CholAU in DMSO was injected, multilamellar and aggregated vesicles were observed after 30 min as shown in Fig. 3B. If all the CholAU molecules were incorporated, this would correspond to about $30 \mathrm{~mol} \%$ of CholAU of total lipid.

Four days after addition of CholAU as a DMSO solution, $\mu \mathrm{m}$ long needle-like structures organized in bundles and aggregates of shorter needles were observed (Fig. 3C and D). This supports our observation that CholAU in the PC membrane was in a metastable state, which matured slowly into the stable needlelike structures.

\section{Decreasing mole fraction of CholAU slows the rate of assembly of the needle-like structures}

We further investigated the morphological changes of lipid vesicles induced by CholAU in GUVs prepared with $\sim 40,20$, and 10 mol\% CholAU by electroformation in the flow chambers. For a $59.2: 40: 0.8$ DMoPC : CholAU : NBD-DMPE mixture, large GUVs were not formed. Only smaller and multilamellar vesicles with diameters of about 1-10 $\mu \mathrm{m}$, pearl necklaces (see insert in Fig. 4A), and aggregates were observed as shown in Fig. 4A. Formation of necklaces upon phase separation in DPPC : POPC : Chol vesicles containing $40 \mathrm{~mol} \%$ of Chol were reported. ${ }^{56}$

For a $79.2: 20: 0.8$ DMoPC : CholAU : NBD-DMPE mixture, large but non-round shaped GUVs were formed (Fig. 4B). Smaller and partly multilamellar vesicles were also observed. Non-round vesicle shape might indicate inhomogeneous
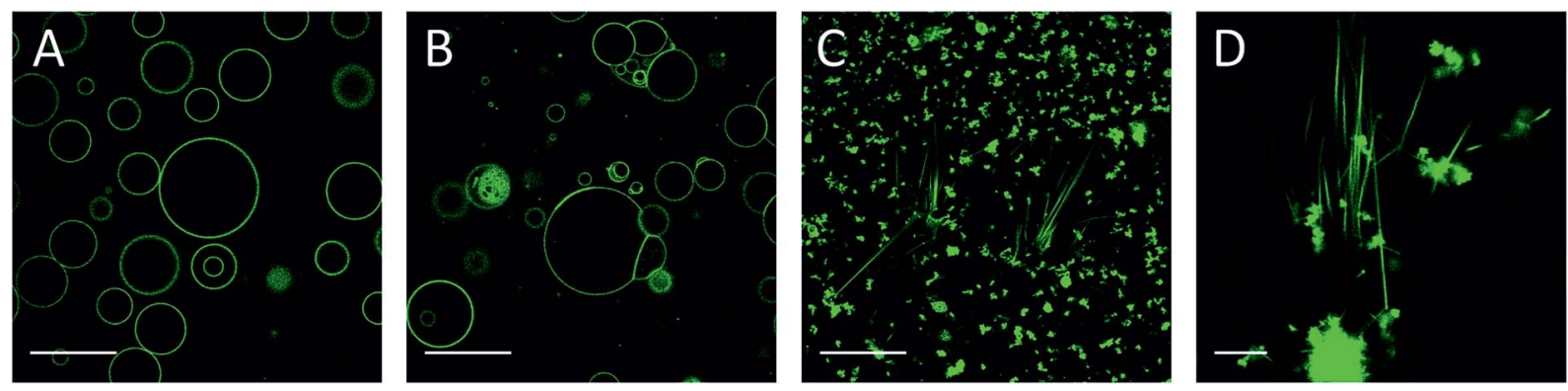

Fig. 3 (a) DMoPC : NBD-DMPE GUVs 1 hour after addition of $20 \mu \mathrm{L}$ DMSO and $580 \mu \mathrm{L}$ water. (b) DMoPC : NBD-DMPE GUVs 30 min after addition of $47 \mu \mathrm{M}$ CholAU in $20 \mu \mathrm{L}$ DMSO and $580 \mu \mathrm{L}$ water. (c) and (d) The same sample as in (b) 4 days after addition of $47 \mu \mathrm{M}$ CholAU in $20 \mu \mathrm{L}$ DMSO and $580 \mu \mathrm{L}$ water. NBD fluorescence images. Scale bars correspond to $50 \mu \mathrm{m}$ for $(\mathrm{a}-\mathrm{c})$ and $10 \mu \mathrm{m}$ for (d). 

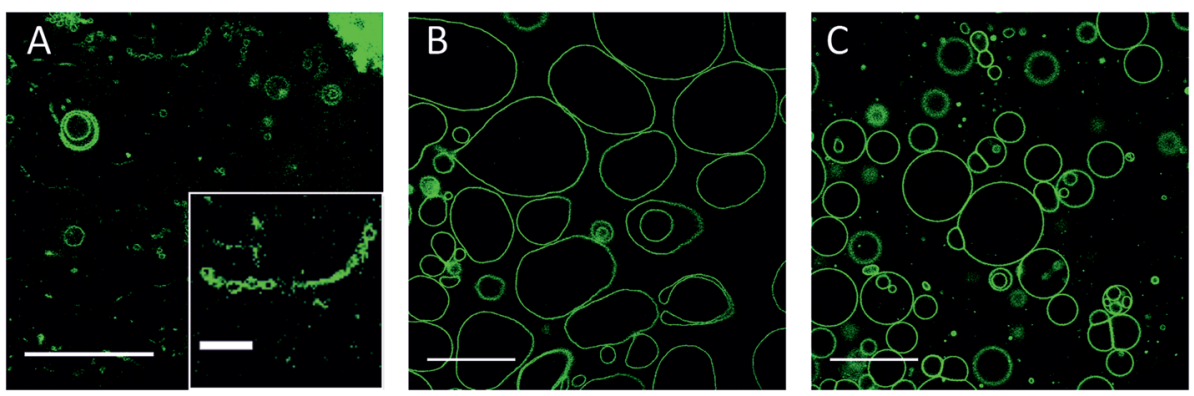

Fig. 4 Morphology of the vesicles formed by electroformation depends on the mole fraction of CholAU in the mixture. NBD fluorescence images of (a) $59.2: 40: 0.8$, (b) $79.2: 20: 0.8$ and (c) $89.2: 10: 0.8$ (molar ratios) DMoPC : CholAU : NBD-DMPE samples, respectively. Scale bars correspond to $50 \mu \mathrm{m}$. Contrast was linearly enhanced. The insert in (a) shows a typical necklace structure observed, scale bar $10 \mu \mathrm{m}$.

distribution of DMOPC and CholAU in the lipid film on the ITO coated glass from which GUVs were prepared. For a $89.2: 10: 0.8$ DMoPC : CholAU : NBD-DMPE mixture, round vesicles with diameters of about $10-40 \mu \mathrm{m}(n=68)$ and aggregates of several (3-10) vesicles as well as much smaller vesicles were observed as shown in Fig. 4C. Similarly to these results, we did not observe giant vesicles for 30 : $70 \mathrm{~mol} \%$ CholAU : DOPC mixture upon hydration at $50{ }^{\circ} \mathrm{C} .{ }^{25}$
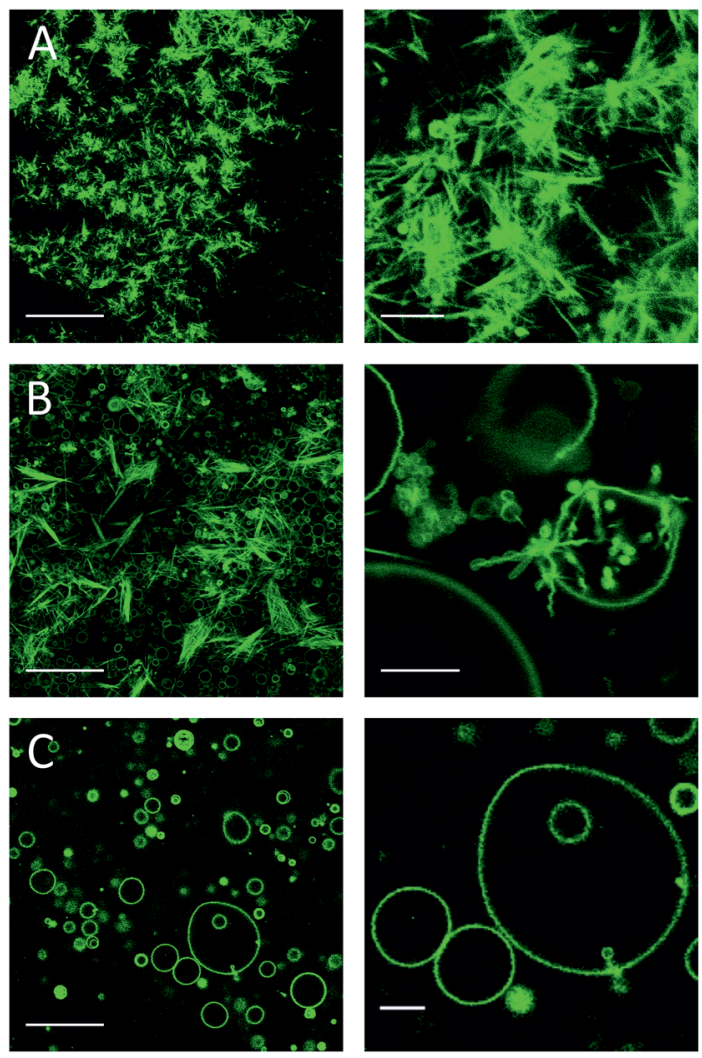

Fig. 5 Needle-like structures formed at different time depending on the mole fraction of CholAU in the mixture. NBD fluorescence images: (a) one day and (b, c) 6 days after preparation of (a) $59.2: 40: 0.8$, (b) $79.2: 20: 0.8$ and (c) $89.2: 10: 0.8$ (molar ratios) DMoPC : CholAU : NBD-DMPE samples, respectively. Scale bars are 50 and $10 \mu \mathrm{m}$ for the images in the right and left panels, respectively.
One day after the preparation, $\mu \mathrm{m}$-long needle-like structures were observed only in the 59.2:40:0.8 DMoPC:ChoIAU:NBD-DMPE sample (Fig. 5A). The clusters of needles were found on the glass surface and in the solution above. For lower mol\% of CholAU, the rate of formation of the needle-like structures was significantly slower: six days after preparation the needle-like structures were observed in the 79.2:20:0.8 DMOPC : CholAU : NBD-DMPE sample, but not yet in the $89.2: 10: 0.8$ DMoPC : CholAU : NBD-DMPE sample as shown in Fig. $5 \mathrm{~B}$ and $\mathrm{C}$. In the latter mixture a few needle-like structures were formed nine days after the preparation (not shown). It should be noted that in the sample with $20 \mathrm{~mol} \%$ of CholAU, vesicle necklaces and needle-like structures covered by lipid tethers were observed even six days after preparation, indicating ongoing assembly (Fig. 5B, right). In line with this observation, non-round shaped GUVs were present in the sample with 10 mol\% of CholAU six days after preparation (Fig. 5C, right panel) indicating an even earlier stage of the assembly process comparable to the vesicle shape changes upon initial incorporation into the pre-formed GUVs membrane. As discussed above, formation of the necklaces and non-round shaped vesicles might be an indication of a phase separation, which presumably occurs before the needle-like structures assemble.

We observed decreased assembly rate with decreased mol\% of CholAU: the slower assembly rate might come from a slower de-mixing of CholAU and DMoPC in a membrane with less CholAU. A slow aggregation of GUVs with $10 \mathrm{~mol} \%$ of CholAU might allow a local enrichment of CholAU in the lipid membrane upon a phase separation. A faster formation of the needle-like structures in the samples containing $30-40 \mathrm{~mol} \%$ (hours) than $10-20 \mathrm{~mol} \%$ (days) indicates that a solubility threshold of CholAU in DMoPC is probably around $40 \mathrm{~mol} \%$.

Most studies on the interaction of Chol with lipid vesicles were performed using max. $30-40 \mathrm{~mol} \% \mathrm{Chol}^{33,35,36,57-59}$ No crystallization was reported upon loading of DOPC GUVs with Chol up to $30-40 \mathrm{~mol} \%$ from $\mathrm{m} \beta \mathrm{CD}-\mathrm{Chol}$ complex. Incorporation of up to $30 \mathrm{~mol} \%$ cholesterol from $10-12 \mathrm{mM}$ Chol- $\mathrm{m} \beta \mathrm{CD}^{51}$ into DOPC vesicles led to $40 \%$ decrease of the diffusion coefficient of a fluorescent lipid analogue indicating an increase of lipid packaging and membrane order, but no separation of optically dense structures from DOPC GUVs was observed. ${ }^{33,39}$ The solubility limit of Chol in 
phospholipids depends on the phospholipids used; threshold of 66-67 mol\% was reported for DOPC and DMPC bilayers. ${ }^{60-65}$ We suppose that incorporation of a higher cholesterol concentration into phosphatidylcholine vesicles would lead to segregation and following precipitation of cholesterol in a form of flat and needle-like structures.

On one hand, the observation that needle-like structures formed promptly when CholAU was delivered using $\mathrm{m} \beta \mathrm{CD}$ suggested that the accumulation of CholAU on one leaflet (i.e. the outer leaflet in this case) might play an important role in the assembly process. The underlying assumption here is that the flip-flop of CholAU is much slower than that of cholesterol, the latter having much smaller polar head and flip-flop halftime of seconds to 1 minute. ${ }^{51,52}$ Accordingly, during the process of tubule formation (30-180 minutes, Fig. 1 and 2), CholAU probably did not have the time to flip and equilibrate through the bilayer. On the other hand, if CholAU was present already during GUV formation, we assumed that the molecule was more evenly distributed between the leaflets (see the low membrane curvature in Fig. $4 \mathrm{~B}, \ll 1 \mu \mathrm{m}^{-1}$ ). In this case, the process of needle formation was indeed $\sim 100$-fold slower.

\section{Needle-like structures are tubules}

We used electron microscopy to study the morphology of the needle-like structures formed. Precipitates were observed on the bottom of the tubes one day after addition of the $2.5 \mathrm{mM}$ mßCD-CholAU complex to DMoPC : NBD-DMPE 99.2: 0.8 (molar ratio) GUVs in 300 mOsm glucose solution. Fluorescence microscopy revealed rosettes of the needle structures as shown in Fig. 6A. For transmission electron microscopy (TEM), the glucose was removed by sedimentation of the needles and resuspension with water; otherwise presence of glucose might impair the visibility of structures (e.g. due to formation of thick and dense films due to the drying and staining process). Aggregates of flat and needle-like structures and single needlelike structures were observed in TEM (Fig. 6B and C). The aggregates resembled rosettes seen in fluorescence microscopy, however, the needles sticking out of the aggregate were significantly shorter. Presumably needles in rosettes were disturbed by the solution exchange and drying procedure. This indicates that weak interactions kept single structures organized into rosettes; this organization was presumably governed by a phospholipid layer (hydrophobic interaction), which would be destroyed upon transfer to the grids and drying. Higher magnification revealed that the needle-like structures represent tubules that withstood drying (Fig. 6C). The tubules had an average outer diameter of 100-200 $\mathrm{nm}$ and a length of 1-3 $\mu \mathrm{m}$ with some tubules being as long as $15 \mu \mathrm{m}(n=96)$. The inner lumen of the tubules partly filled with the contrast agent had on average diameters of 15-50 nm. Box plots of the tubules dimensions are shown in ESI. $\dagger$

Differential interference contrast (DIC) images revealed optically dense needle-like structures (Fig. 2C). In TEM we observed that the structures were tubules with dense walls of 50-100 nm thickness, which explained the high contrast in DIC. The tubules withstood air drying as seen before for $\sim 300 \mathrm{~nm}$ diameter CholAU-DOPC tubules formed upon cooling from 70 ${ }^{\circ} \mathrm{C} .{ }^{25}$ The tubules were enriched in CholAU containing up to 90 mol\% of CholAU and covered by a phospholipid membrane in a fluid crystalline state. ${ }^{25}$ Here, again homogeneous distribution of the NBD-DMPE fluorescence in the needle-like structures was observed indicating that the structures were probably covered by a phospholipid layer. Phosphatidylcholine plays multiple roles in supporting the assembly of tubules. First, the phospholipid is a solvent for CholAU preventing precipitation of the pure conjugate in aqueous solutions. Similarly, solubility of Chol in human bile depends on the PC structure and Chol-PC ratio; precipitation of Chol with transformation through helices and tubules to flat monohydrous crystals were observed for Chol super-saturated bile. ${ }^{66-68}$ Second, lipid bilayers serve as a platform (two-dimensional phase) for the initial de-mixing of CholAU. Taking a parallel to Chol again, separation of Chol into clusters was reported for concentrations exceeding the saturation threshold..$^{61,65,69}$ Third, phospholipid vesicles changing upon formation of the intermediate aggregates, provide the necessary environment for tubular formation, which otherwise often can be seen only in organic solvents. Formation of multilamellar structures might provide the necessary environment for further self-assembly. We previously observed that CholAUDOPC tubules were formed only inside lipid vesicles aggregates. ${ }^{25}$ It was reported that nucleation of the cholesterol crystal growth in bile was initiated upon formation of multilamellar vesicles. ${ }^{18}$ Fourth, the tubules are covered by a fluid layer of lipids ${ }^{25}$ which presumably stabilizes the tubules in the aqueous solution providing a hydrophilic surface.
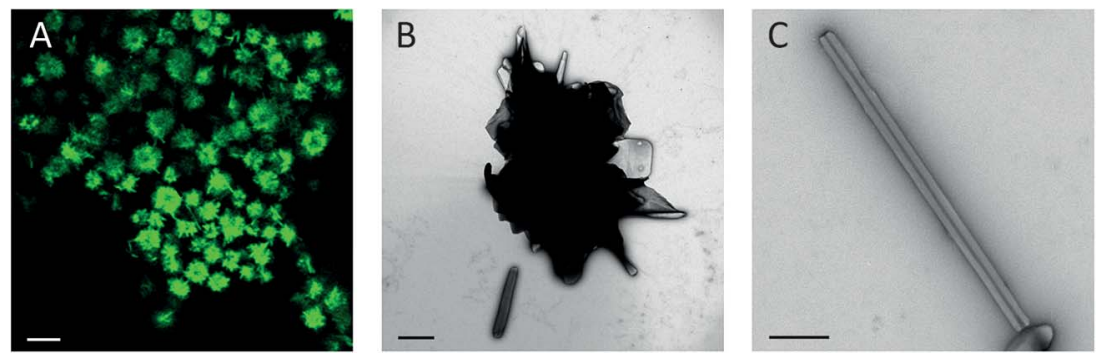

Fig. 6 (a) NBD fluorescence image of the needle-like structures organized in rosettes 20 hours after addition of $2.5 \mathrm{mM}$ of $\mathrm{m} \beta C D-C h o l A U$ complex to DMoPC : NBD-DMPE GUVs. Scale bar corresponds to $10 \mu \mathrm{m}$. (b) TEM image of a rosette-like structure with tubules. (c) TEM image of a single tubule. Scale bars correspond to $1 \mu \mathrm{m}$ and $500 \mathrm{~nm}$ for (b) and (c), respectively. 
The self-assembled structures in the phospholipid-CholAU binary system resemble microscopically the structures reported for cholesterol self-assembly from oversaturated bile solutions. Comparing our findings to the reported for cholesterol assembly we might get further insight into the properties of the system, even though CholAU needle-like structures formed in the presence of phospholipid bilayers, whereas cholesterol assembly occurs from oversaturated mixed micelles. The needle-like structures formed from cholesterol in bile solution are thought to be anhydrous or a mixture of anhydrous and monohydrous Chol crystals covered by one layer of phosphatidylcholine. ${ }^{6,59,70}$ Needle-like structures are considered to be a precursor for further cholesterol crystallization through helices and tubules ending with flat crystals of monohydrous Chol. ${ }^{67,71}$ In contrast, we observed that both CholAU-PC tubules and flat crystals were stable for several months. We do not have enough evidence yet to report the state of CholAU in the structures, whereas we have observed that phospholipids coating the tubules were in a fluid liquid state. ${ }^{25}$ Finally, the assembly happened at lower mole fraction of CholAU $(<40 \mathrm{~mol} \%)$ than the Chol saturation threshold $(67 \mathrm{~mol} \%)$ suggesting the important role for the uridine head group for the self-assembly of CholAU.

One other parameter that can be compared is the time needed for the needle structure assembly: cholesterol needles were formed within weeks, while CholAU needles were formed within hours to days. This is in line with the difference in the rate of micrometer sized tubules formation: cholesterol microtubules in bile salt were reported to form in about 3-6 days and were unstable; ${ }^{6}$ CholAU-DOPC microtubules formed within minutes upon cooling from $70{ }^{\circ} \mathrm{C}$ to room temperature and were stable for months. ${ }^{25}$

We surmise that the differences in CholAU and Chol selfassembly might be due to the additional self-aggregating properties of the uridine head group of CholAU (stacking and hydrogen bonding) in comparison with just one hydrogen bond that could be formed by the OH group of Chol.

\section{Conclusions}

We have previously reported the self-assembly of CholAU with a phospholipid into nano- and microtubules containing about 80-90 mol\% of CholAU starting from $30: 70$ CholAU : DOPC (molar ratio) multilamellar vesicles. ${ }^{25}$ Incubation of the CholAU : DOPC mixture at $70{ }^{\circ} \mathrm{C}$, presumably supporting the solubilization of CholAU in the DOPC membrane, and cooling to room temperature was reported to be essential for the formation of the tubular structures. Here we report that the nanotubules were formed even at room temperature when using solubilized CholAU and DMoPC giant unilamellar vesicles, indicating that the limiting factor is indeed the degree of incorporation of the conjugate into the lipid bilayer (the self-segregation and precipitation of CholAU). Different methods of CholAU addition were tested: incubation of pre-formed DMoPC : NBD-DMPC GUVs in flow chambers with 2.5 $\mathrm{mM} \mathrm{m} \beta \mathrm{CD}-\mathrm{CholAU}$; incubation of the GUVs in flow chambers with CholAU dissolved in DMSO; electroformation of GUVs from DMoPC : CholAU : NBD-DMPC mixtures in flow chambers; addition of $2.5 \mathrm{mM} \mathrm{m} \beta \mathrm{CD}-\mathrm{CholAU}$ to DMoPC : NBD-DMPC GUVs in plastic tubes. The needle-like structures were formed in all samples when CholAU was present, although the rate of the formation strongly depended on the molar ratio of CholAU to total lipid in the sample. Moreover, we observed that the structures formed more promptly when CholAU was incorporated into the outer leaflet of the membrane, suggesting an intrinsic curvature effect due to the incorporation of the molecules. Transmission electron microscopy revealed that the needle-like structures represent tubules of 100-200 nm outer diameters and dense walls of 50-100 nm thickness. The inner lumen diameter of the tubules was in average $15-40 \mathrm{~nm}$.

Our results reveal that nanometer sized tubules are intrinsic and stable structures formed in the binary system of cholesterylaminouridine and phosphatidylcholine even at room temperature. The novel assembly process described in this study opens the possibility to form such nanotubules in the presence of biological macromolecules for which high temperatures might be deleterious. Synthesis of cholesteryl nucleoside conjugates with other nucleobases for investigation of their ability to form nanotubules under similar conditions is presently underway in our laboratories.

\section{Acknowledgements}

This work was supported by the grant of the Deutsche Forschungsgemeinschaft, DFG, AR 783/1-1 to AA.

\section{References}

1 G. M. Whitesides and B. Grzybowski, Science, 2002, 295, 2418-2421.

2 J.-M. Lehn, Proc. Natl. Acad. Sci. U. S. A., 2002, 99, 4763-4768. 3 J. N. Israelachvili, D. J. Mitchell and B. W. Ninham, J. Chem. Soc., Faraday Trans. 2, 1976, 72, 1525-1568.

4 P. R. Cullis and B. De Kruijff, Biochim. Biophys. Acta, 1979, 559, 399-420.

5 A.-L. Fameau, F. Cousin, L. Navailles, F. Nallet, F. Boué and J.-P. Douliez, J. Phys. Chem. B, 2011, 115, 9033-9039.

6 F. M. Konikoff, D. S. Chung, J. M. Donovan, D. M. Small and M. C. Carey, J. Clin. Invest., 1992, 90, 1155-1160.

7 G. S. Abela, J. Clin. Lipidol., 2010, 4, 156-164.

8 J. H. Jung, M. Park and S. Shinkai, Chem. Soc. Rev., 2010, 39, 4286-4302.

9 N. Kameta, H. Minamikawa and M. Masuda, Soft Matter, 2011, 7, 4539-4561.

10 T. Shimizu, M. Masuda and H. Minamikawa, Chem. Rev., 2005, 105, 1401-1443.

11 A. Bot and W. G. M. Agterof, JAOCS, 2006, 83, 513-521.

12 S. J. Lee, E. Kim, M. L. Seo, Y. Do, Y.-A. Lee, S. S. Lee, J. H. Jung, M. Kogiso and T. Shimizu, Tetrahedron, 2008, 64, 1301-1308.

13 J. V. Trillo, F. Meijide, A. Jover, V. H. Soto, S. de Frutos, M. Chiara di Gregorio, L. Galantini and J. V. Tato, $R S C$ Adv., 2014, 4, 5598-5606.

14 Z. Shen, T. Wang and M. Liu, Chem. Commun., 2014, 50, 2096-2099. 
15 D. Berti, F. B. Bombelli, M. Fortini and P. Baglioni, J. Phys. Chem. B, 2007, 111, 11734-11744.

16 C. Aimé, R. Tamoto, T. Satoh, A. Grelard, E. J. Dufourc, T. Buffeteau, H. Ihara and R. Oda, Langmuir, 2009, 25, 8489-8496.

17 Y. V. Zastavker, N. Asherie, A. Lomakin, J. Pande, J. M. Donovan, J. M. Schnur and G. B. Benedek, Proc. Natl. Acad. Sci. U. S. A., 1999, 96, 7883-7887.

18 D. Q.-H. Wang, D. E. Cohen and M. C. Carey, J. Lipid Res., 2009, S406-S411.

19 B. Jean, L. Oss-Ronen, P. Terech and Y. Talmon, Adv. Mater., 2005, 17, 728-731.

20 X. Zhang, J. Zou, K. Tamhane, F. F. Kobzeff and J. Fang, Small, 2010, 6, 217-220.

21 V. H. Soto Tellini, A. Jover, F. Meijide, J. Vázquez Tato, L. Galantini and N. V. Pavel, Adv. Mater., 2007, 19, 17521756.

22 L. Galantini, C. Leggio, A. Jover, F. Meijide, N. V. Pavel, V. H. Soto Tellini, J. V. Tato, R. Di Leonardo and G. Ruocco, Soft Matter, 2009, 5, 3018-3025.

23 E. Snip, K. Koumoto and S. Shinkai, Tetrahedron, 2002, 58, 8863-8873.

24 Y. Jin, R. Xin, P. Ai and D. Chen, Int. J. Pharm., 2008, 350, 330-337.

25 P. Pescador, N. Brodersen, H. A. Scheidt, M. Loew, G. Holland, N. Bannert, J. Liebscher, A. Herrmann, D. Huster and A. Arbuzova, Chem. Commun., 2010, 46, 5358-5360.

26 O. Wesołowska, K. Michalak, J. Maniewska and A. B. Hendrich, Acta Biochim. Pol., 2009, 56, 33-39.

27 P. Walde, K. Cosentino, H. Engel and P. Stano, ChemBioChem, 2010, 11, 848-865.

28 S. Chiantia, J. Ries and P. Schwille, Biochim. Biophys. Acta, 2009, 1788, 225-233.

29 J. M. Holopainen, M. I. Angelova and P. K. Kinnunen, Biophys. J., 2000, 78, 830-838.

30 B. Sorre, A. Callan-Jones, J. Manzi, B. Goud, J. Prost, P. Bassereau and A. Roux, Proc. Natl. Acad. Sci. U. S. A., 2012, 109, 173-178.

31 M. Safouane, L. Berland, A. Callan-Jones, B. Sorre, W. Römer, L. Johannes, G. E. S. Toombes and P. Bassereau, Traffic, 2010, 11, 1519-1529.

32 N. Kahya, D. Scherfeld, K. Bacia and P. Schwille, J. Struct. Biol., 2004, 147, 77-89.

33 N. Kahya, D. Scherfeld, K. Bacia, B. Poolman and P. Schwille, J. Biol. Chem., 2003, 278, 28109-28115.

34 D. Scherfeld, N. Kahya and P. Schwille, Biophys. J., 2003, 85, 3758-3768.

35 M. Loew, R. Springer, S. Scolari, F. Altenbrunn, O. Seitz, J. Liebscher, D. Huster, A. Herrmann and A. Arbuzova, J. Am. Chem. Soc., 2010, 132, 16066-16072.

36 M. Schade, A. Knoll, A. Vogel, O. Seitz, J. Liebscher, D. Huster, A. Herrmann and A. Arbuzova, J. Am. Chem. Soc., 2012, 134, 20490-20497.

37 T. Baumgart, G. Hunt, E. R. Farkas, W. W. Webb and G. W. Feigenson, Biochim. Biophys. Acta, 2007, 1768, 21822194.
38 M. I. Angelova and D. S. Dimitrov, Faraday Discuss., 1986, 81, 303-311.

39 S. Chiantia, P. Schwille, A. S. Klymchenko and E. London, Biophys. J., 2011, 100, L01-L03.

40 H.-T. Cheng, Megha and E. London, J. Biol. Chem., 2009, 284, 6079-6092.

41 J. Yuan, S. M. Hira, G. F. Strouse and L. S. Hirst, J. Am. Chem. Soc., 2008, 130, 2067-2072.

42 M. H. Mohamed, L. D. Wilson, J. V Headley and K. M. Peru, Rapid Commun. Mass Spectrom., 2009, 23, 3703-3712.

43 C. A. López, A. H. de Vries and S. J. Marrink, PLoS Comput. Biol., 2011, 7, e1002020.

44 S. Milles, T. Meyer, H. A. Scheidt, R. Schwarzer, L. Thomas, M. Marek, L. Szente, R. Bittman, A. Herrmann, T. Günther Pomorski, D. Huster and P. Müller, Biochim. Biophys. Acta, 2013, 1828, 1822-1828.

45 S. Grimmer, M. Ying, B. Van Deurs and K. Sandvig, Traffic, 2005, 6, 144-156.

46 A.-F. Bitbol, N. Puff, Y. Sakuma, M. Imai, J.-B. Fournier and M. I. Angelova, Soft Matter, 2012, 8, 6073-6082.

47 A. Papadopulos, S. Vehring, I. López-Montero, L. Kutschenko, M. Stöckl, P. F. Devaux, M. Kozlov, T. Pomorski and A. Herrmann, J. Biol. Chem., 2007, 282, 15559-15568.

48 U. Seifert, K. Berndl and R. Lipowsky, Phys. Rev. A, 1991, 44, 1182-1202.

49 T. Tanaka, R. Sano, Y. Yamashita and M. Yamazaki, Langmuir, 2004, 20, 9526-9534.

50 Y. Li, R. Lipowsky and R. Dimova, Proc. Natl. Acad. Sci. U. S. A., 2011, 108, 4731-4736.

51 R. Leventis and J. R. Silvius, Biophys. J., 2001, 81, 2257-2267.

52 K. John, J. Kubelt, P. Müller, D. Wüstner and A. Herrmann, Biophys. J., 2002, 83, 1525-1534.

53 R. Zidovetzki and I. Levitan, Biochim. Biophys. Acta, 2007, 1768, 1311-1324.

54 Y. Yamashita, M. Oka, T. Tanaka and M. Yamazaki, Biochim. Biophys. Acta, 2002, 1561, 129-134.

55 Y. Sun, C.-C. Lee, W.-C. Hung, F.-Y. Chen, M.-T. Lee and H. W. Huang, Biophys. J., 2008, 95, 2318-2324.

56 M. Yanagisawa, M. Imai and T. Taniguchi, Phys. Rev. E, 2010, 82, 051928.

57 I. Visco, S. Chiantia and P. Schwille, Langmuir, 2014, 30, 7475-7484.

58 I. Levental, M. Grzybek and K. Simons, Proc. Natl. Acad. Sci. U. S. A., 2011, 108, 11411-11416.

59 E. Baykal-Caglar, E. Hassan-Zadeh, B. Saremi and J. Huang, Biochim. Biophys. Acta, 2012, 1818, 2598-2604.

60 J. Huang, J. T. Buboltz and G. W. Feigenson, Biochim. Biophys. Acta, 1999, 1417, 89-100.

61 L. Mainali, M. Raguz and W. K. Subczynski, J. Phys. Chem. B, 2013, 117, 8994-9003.

62 R. M. Epand, R. F. Epand, D. W. Hughes, B. G. Sayer, N. Borochov, D. Bach and E. Wachtel, Chem. Phys. Lipids, 2005, 135, 39-53.

63 D. Marsh, Biochim. Biophys. Acta, 2010, 1798, 688-699. 
64 M. A. Barrett, S. Zheng, L. A. Toppozini, R. J. Alsop, H. Dies, A. Wang, N. Jago, M. Moore and M. C. Rheinstädter, Soft Matter, 2013, 9, 9342-9351.

65 R. Ziblat, I. Fargion, L. Leiserowitz and L. Addadi, Biophys. J., 2012, 103, 255-264.

66 M. C. Frincu, S. D. Fleming, A. L. Rohl and J. A. Swift, J. Am. Chem. Soc., 2004, 126, 7915-7924.
67 P. Portincasa, A. Moschetta, K. J. van Erpecum, G. Calamita, A. Margari, G. P. VanBerge-Henegouwen and G. Palasciano, Dig. Liver Dis., 2003, 35, 118-126.

68 L. Hosta-Rigau, Y. Zhang, B. M. Teo, A. Postma and B. Städler, Nanoscale, 2013, 5, 89-109.

69 J. Huang and G. W. Feigenson, Biophys. J., 1999, 76, 21422157.

70 R. Abendan and J. Swift, Langmuir, 2002, 11, 4847-4853.

71 D. Q. Wang and M. C. Carey, J. Lipid Res., 1996, 37, 606-630. 